\title{
Análise da atuação das enfermeiras em um modelo de unidades gerenciais
}

\section{Nursery performance analysis within a model of management units}

\author{
Maria Luiza Hexsel Segui ${ }^{1}$; Aida Maris Peres ${ }^{2}$; Elizabeth Bernardino ${ }^{3}$
}

\begin{abstract}
Resumo
Objetivo: identificar os espaços de atuação das enfermeiras após a implantação do modelo de Unidades Gerenciais em uma instituição hospitalar de ensino de grande porte e alta complexidade, da região sul do Brasil. Método: estudo exploratório descritivo de abordagem qualitativa, na modalidade de estudo de caso. Os dados foram coletados por entrevista semi-estruturada com 15 enfermeiras representantes dos cargos ocupados pela categoria na instituição. Resultados: as participantes são do sexo feminino, de 25 a 59 anos, com tempo de atuação no hospital entre 05 e 30 anos, a maioria pós-graduada. As atividades dividem-se em: assessoria em comissões ou comitês, vigilância em saúde, atividades de gerenciamento, assistência de enfermagem, educação em serviço, orientação em saúde, pesquisa e atividades administrativas institucionais. Conclusões: as enfermeiras que trabalham com assessoria sustentam a operacionalização de algumas políticas públicas de saúde; nos cargos da estrutura do Serviço de Enfermagem, atividades estão relacionadas ao gerenciamento do cuidado e da unidade; e nos cargos administrativos externos, visam às demandas institucionais gerais.
\end{abstract}

Palavras-chave: Enfermagem. Prática profissional. Gerenciamento da prática profissional.

\begin{abstract}
Objective: Identify the nursery's working spaces after implementation of a Unit Management model at a large and complex teaching hospital in south of Brazil. Method: exploratory descriptive study with a qualitative approach - Case Study. The data were collected with semi-structured interviews. Subjects: 15 nurses that represent the job positions at the institution. Results: All subjects are female, aged from 25 to 59 years, with high-level education, work experience at the hospital between 5 and 30 years, most of them have a postgraduate degree. The activities are divided on consulting services, public health surveillance, management, nursing care, on-the-job education, health education, research, and university-related management work. Conclusion: nurses working as assistants give support to public health surveillance; at Nursing Services, the activities are related to unit and care management; and at external administrative positions, they support general institutional demands.
\end{abstract}

Key words: Nursing. Professional practice. Practice management.

\footnotetext{
${ }^{1}$ Enfermeira, Mestre em Enfermagem da Universidade Federal do Paraná. Enfermeira do HC/UFPR. Email: segui@ufpr.br

${ }^{2}$ Doutora em Enfermagem pela Universidade de São Paulo. Professora Adjunta do Departamento de Enfermagem da Universidade Federal do Paraná. Email: amaris@ufpr.br

${ }^{3}$ Doutora em Enfermagem pela EERP/USP. Professora Adjunta do Curso de Enfermagem da UFPR. Professora Associada da Universitá Laval, Quebec, Canadá. Email: elizaber@ufpr.br
} 


\section{Introdução}

A implantação do Sistema Único de Saúde (SUS), as transformações sociais e econômicas, usuários cada vez mais exigentes, a crescente incorporação de tecnologias de toda natureza, impulsionam os dirigentes hospitalares a buscar estratégias gerenciais compatíveis a este cenário, uma vez que o modelo administrativo tradicional não atende mais estas demandas.

Foi assim que em 2002, a direção do hospital em estudo efetuou alterações significativas no seu gerenciamento, adotando o modelo teórico de linha de cuidado, entendido como o somatório de um grande número de pequenos cuidados parciais que o usuário recebe e que vão se complementando, conforme as suas necessidades, passando por serviços de apoio diagnóstico e terapêutico, especialidades, atenção hospitalar e outros (CECÍLIO; MERHY, 2003). O modelo foi implantado institucionalmente com a criação de unidades administrativas ou assistenciais inicialmente denominadas Unidades Funcionais e após Unidades Gerenciais (UG).

O modelo gerencial de UG caracteriza-se pela descentralização e democratização das decisões, resolução mais rápida e desburocratizada dos problemas cotidianos do hospital, possibilitando a comunicação interna mais ágil entre os gerentes das UG (CECÍLIO, 2009; CECÍLIO; MERHY, 2003).

Este hospital define UG como o resultado da agregação de serviços/atividades e/ou especialidades cujas afinidades articuladas estabelecem uma linha de cuidado, visando o atendimento integral e de qualidade ao usuário. São estabelecidos contratos/pactos internos que possibilitem um melhor desempenho de toda a equipe, evitando-se a fragmentação do cuidado ou serviço, promovendo o gerenciamento mais eficaz da instituição (UNIVERSIDADE FEDERAL DO PARANÁ, 2002).

O modelo gerencial adotado implicou em transformações no processo de trabalho dos profissionais. Segundo Paganini et al. (2000), os mais afetados pelas mudanças foram os enfermeiros, que antes desempenhavam principalmente funções administrativas, restritas ao processo de trabalho da categoria de enfermagem e a funções assistenciais, participavam em comissões, como controle de infecção hospitalar e de compras. Já com o novo desenho organizacional, as enfermeiras começaram a ocupar cargos externos à estrutura do serviço de enfermagem, possibilitando uma ampliação de suas funções.

Considerando que esta nova configuração gerencial permitiu às enfermeiras ampliar sua atuação para além dos espaços tradicionalmente ocupados por elas, pergunta-se: quais são os espaços de atuação ocupados pelas enfermeiras neste hospital após a implantação do modelo de unidades funcionais?

Assim, este artigo tem como objetivo identificar os espaços de atuação das enfermeiras em uma instituição hospitalar de ensino após a implantação do modelo de Unidades Gerenciais.

\section{Método}

Esta é uma pesquisa exploratória descritiva, de abordagem qualitativa na modalidade de estudo de caso, que teve como campo de estudo um hospital de ensino de grande porte e alta complexidade, na cidade de Curitiba, PR, onde atuam 1.121 profissionais de enfermagem, dos quais 222 enfermeiros, 186 técnicos de Enfermagem e 713 auxiliares de Enfermagem (UNIVERSIDADE FEDERAL DO PARANÁ, 2011).

Para definir os participantes da coleta de dados da pesquisa, obteve-se uma listagem contendo o nome das 222 enfermeiras que trabalhavam no hospital e seus respectivos cargos, sendo que sete delas encontravam-se afastadas. Assim, o universo pesquisado foi de 215 enfermeiras. Buscando-se a representatividade dos diversos cargos exercidos pelas enfermeiras na instituição, foi necessário fazer uma primeira seleção por aproximação 
de cargos, e desta emergiram três categorias: enfermeiras que ocupam cargos permanentes em comissões; enfermeiras que ocupam cargos dentro da estrutura do Serviço de Enfermagem e enfermeiras que ocupam cargos gerais externos ao Serviço de Enfermagem. Após esta identificação e categorização foi definida de forma intencional que cada cargo teria um participante representado na amostra do estudo, considerando-se que estes permitem reconhecer em sua diversidade, o trabalho da enfermeira no cenário de estudo.

Assim, a amostra foi composta por: 04 enfermeiras de comissão/comitê; 08 enfermeiras dos cargos dentro da estrutura do Serviço de Enfermagem, sendo quatro representantes dos serviços que já fazem parte de UG e 04 dos serviços que ainda não constituíam UG; e 03 enfermeiras que ocupam cargos externos ao Serviço de Enfermagem, totalizando 15 participantes, escolhidos por sorteio. Todas as sorteadas concordaram em participar da entrevista.

A coleta de dados foi realizada por meio de entrevista semi-estruturada (MINAYO; DESLANDES; GOMES, 2008) combinando questões abertas e fechadas, durante os meses de fevereiro e março de 2010, no ambiente de trabalho dos participantes, com duração média de quarenta e cinco minutos. As entrevistas foram gravadas e posteriormente transcritas. $\mathrm{O}$ instrumento utilizado foi submetido a teste piloto e, após este, ajustado conforme as necessidades identificadas.

Os dados foram analisados pela técnica de análise de conteúdo (BARDIN, 2011) seguindo três etapas: a pré-análise; a exploração do material e o tratamento dos resultados; a inferência e a interpretação.

O projeto foi aprovado pelo Comitê de Ética em Pesquisa do Setor de Ciências da Saúde/UFPR e teve como Certificado de Apresentação para Apreciação Ética (CAAE), o n ${ }^{\circ}$ 0060.091.000-09. Para garantir o anonimato das enfermeiras entrevistadas, elas foram identificadas pela letra $\mathrm{E}$ e um número arábico escolhido de acordo com a sequência em que as entrevistas foram realizadas.

\section{Resultados}

Todas as participantes do estudo eram do sexo feminino, com idade entre 25 e 59 anos, sendo 13 com mais de 40 anos. Quanto ao tempo de formação acadêmica, $26,7 \%$ das enfermeiras estavam formadas há mais de 20 anos e 46,7\% terminaram a graduação há mais de 25 anos. O levantamento do tempo de atuação na instituição indicou que $66,7 \%$ dos participantes da pesquisa trabalhavam no hospital entre 15 e 30 anos, e 14 enfermeiras concluíram ou realizavam cursos de pós-graduação. Destas, quatro eram mestres, três mestrandas e duas doutorandas.

\section{Cargos de comissão/comitê}

Os cargos de comissão/comitê e assessoria são ocupados por um ou mais profissionais especializados em uma determinada área de conhecimento, Estes elaboram metodologias e desenvolvem padrões de trabalho, além da criação de protocolos, abrangendo várias áreas do hospital em decorrência de necessidades da administração, da assistência ao paciente e de programas em curso na instituição. Também são responsáveis pela busca de soluções para problemas e/ ou necessidades pontuais internas ou intra-áreas.

As comissões/comitês são normativas, não têm subordinados, trabalham em parceria, cooperação e colaboração com outros profissionais, que colocam em prática as instruções destas.

Neste estudo foram considerados apenas as comissões/comitês com enfermeiras que trabalham com exclusividade nestes, representados pelo Comitê de Acreditação, Serviço de Epidemiologia, Comissão de Controle de Infecção Hospitalar e Comitê Transfusional. Também assessoram a Direção Geral na tomada de decisões que correspondem as suas áreas de conhecimento. 
Para os cargos de comissão/comitê, no geral, o número de enfermeiras é definido por legislação específica, critério observado pelo hospital. São elas que operacionalizam algumas importantes políticas públicas de saúde, relacionadas ao controle de infecção hospitalar, aos hemoderivados, ao serviço de epidemiologia e a qualidade do serviço prestado pelo hospital.

A atuação das enfermeiras acontece no âmbito do planejamento das ações pertinentes a cada área, na educação em serviço visando tornar público e uniformizar as normas estabelecidas para os diferentes serviços do hospital e no controle do seguimento das mesmas.

As atividades das enfermeiras em comissões/ comitês foram caracterizadas como assessoria, vigilância em saúde e educação em serviço. Dentre elas fazem parte o diagnóstico de situação, planejamento das ações a serem desenvolvidas, que são repassadas às enfermeiras e equipe multiprofissional dos serviços do hospital, através de educação em serviço para que estas ações sejam colocadas em prática. A efetivação ou não destas ações, ou seja, os resultados são divulgados em reuniões informativas e boletins técnicos.

Como era um setor novo, o principal trabalho

foi estruturar e desenvolver uma tecnologia para implantar o processo. [...] um trabalho de coordenar a equipe, e um trabalho de, tinha que fazer relatório também, estabelecer uma forma de devolução para as equipes que foram auditadas. (E6)

A vigilância em saúde está representada nestas falas:

As atividades são muito dinâmicas porque o que acontece nas unidades você já tem que fazer uma ação. Eu analiso e vou discutir com a chefia, vou apresentar isso para a chefia [...]. E o que o comitê faz, ele reforça que tem rotinas que precisam ser cumpridas. (E14)

A educação em serviço é uma atividade com grande significância para estas enfermeiras e são direcionadas não apenas para a enfermagem, mas também para a equipe multiprofissional. Nós temos que ter planejamento das atividades educativas, [...] nós somos muito convidados para dar aulas, palestras, para profissionais tanto do nível médio como do superior. (E14)

Nos depoimentos das entrevistadas fica evidenciada a necessidade da elaboração de um planejamento para o trabalho a ser desenvolvido, a execução das ações planejadas para alcançar o objetivo pretendido e a divulgação dos resultados para a avaliação dos mesmos.

\section{Cargos dentro da estrutura do Serviço de Enfermagem}

Estes são ocupados por enfermeiras e são denominados de Enfermeira Assistencial, Enfermeira Administrativa (chefia de serviço), Supervisão de Enfermagem de UG, Coordenação de Enfermagem, Assessoria de Enfermagem e Supervisão Geral de Enfermagem. Estas têm como subordinados enfermeiras, técnicos e auxiliares de Enfermagem.

Para os cargos de enfermagem, as atividades foram divididas em atividades administrativas, atividades assistenciais, educação em serviço, orientação em saúde e pesquisa.

As atividades administrativas compreendem o planejamento da infraestrutura necessária ao desenvolvimento do trabalho de enfermagem e da equipe multidisciplinar, o diagnóstico das necessidades para a execução da assistência de enfermagem, a previsão e controle de recursos humanos na elaboração e controle das escalas de trabalho e de atividades, fechamento de folha-ponto e horas extras; a previsão e controle de recursos materiais e de equipamentos, e manutenção preventiva ou corretiva destes; a resolução de conflitos entre a equipe, paciente e familiar.

As atividades administrativas são realizadas com a finalidade de assegurar a qualidade da assistência de enfermagem e o bom funcionamento do serviço. 
[...] desde a organização da escala de trabalho, de atividades, organização da assistência, relativos a fechamento de folha ponto, horas extras. (E1)

O gerenciamento dos recursos materiais e equipamentos dos serviços de saúde foram citados pelas enfermeiras como atividade administrativa.

Se falta algum material eu vou atrás, manutenção de equipamento, sempre dou uma controlada. (E2)

Gerenciar conflitos também compõe o cotidiano do trabalho gerencial administrativo da enfermeira:

Problemas de relacionamento na equipe, alguns problemas de relacionamento com pacientes e familiares e equipe. (E3)

Nas atividades assistenciais as enfermeiras enfatizam o planejamento dos cuidados diários de enfermagem, o cuidado direto ao paciente e a execução de cuidados de enfermagem de maior complexidade técnica. A enfermeira tem como objeto de intervenção as necessidades de cuidado de enfermagem, que se dá através da articulação entre a ação de planejamento da assistência e a ação de cuidado direto acompanhado da interação e do vínculo com o paciente.

Faço os cuidados integrais dos pacientes daquele dia. (E4)

As falas das enfermeiras demonstram que a educação em serviço tem um caráter esporádico, não é incorporada no cotidiano como uma função da enfermeira.

Alguma educação em serviço sobre uma PAM (pressão arterial média), um ventilador novo, uma bomba infusora nova. Mas é uma coisa de momento, não planejado no semestre. (E10)

Como hospital de ensino, as enfermeiras realizam o acompanhamento de acadêmicos e estagiários:

Acompanhamento de acadêmicos do $9^{\circ}$ período da Enfermagem. (E1)

A orientação em saúde é realizada pela enfermeira para o paciente e familiar principalmente no momento da alta hospitalar.
Oriento os pais, dou informações, orientação para as pacientes. (E2)

Uma das enfermeiras referiu a pesquisa como parte das atividades cotidianas:

Organização de pesquisa dentro da unidade e grupo de estudo. (E1)

\section{Cargos gerais externos ao Serviço de Enfermagem}

Estes podem ser ocupados por qualquer profissional de nível superior. Neste estudo foram considerados aqueles que no período da coleta de dados estavam sendo ocupados por enfermeiras: Direção de Assistência, gerências de Unidade Gerencial e serviços administrativos, estratégicos para a instituição, como a Coordenação de Internação, Unidade de Abastecimento e Serviço de Faturamento.

Estas enfermeiras trabalham em atividades administrativas no nível institucional, que abrangem planejamento e gerenciamento das metas a serem atingidas, controle dos leitos, faturamento, previsão de recursos humanos, previsão e controle de recursos materiais, aquisição de equipamentos, resolução de problemas/conflitos multiprofissionais e institucionais. O trabalho realizado de coordenação compartilhada e de cooperação entre diferentes profissionais é uma característica encontrada no modelo administrativo adotado na instituição desde 2002.

Planejamento, trabalho muito em cima de planejamento, é planejamento das atividades mesmo, que incluem os programas de acreditação, humanização, as metas, gerenciamento de recursos humanos, gerenciamento de recursos materiais. (E9)

É toda parte administrativa relacionada aos internamentos, aos leitos e transferências, controle dos transplantes [...]. (E7)

Esta fala permite inferir que as decisões macro institucionais acontecem no âmbito da direção superior colegiada, considerando o hospital como 
um todo, deixando as específicas para os colegiados das UG, representando a dinâmica do modelo gerencial vigente:

Então este gerenciamento com as UF é feito através do gerente, [...] despacho semanal com cada gerente, a gente toma as decisões macro e aí no micro tudo é levado para o colegiado, [...]. Com a implantação das UF facilitou muito o trabalho no operacional, porque o gerente faz esta ponte [...]. (E15)

\section{Discussão}

O hospital em estudo tem caráter público e, como tal, os servidores possuem estabilidade no trabalho, garantida pela política pública vigente, o que leva o profissional a permanecer na instituição.

Os participantes representam um grupo titulado, convergindo com a compreensão de que a educação permanente consiste em um compromisso de desenvolvimento pessoal, uma vez que o processo de globalização exige contínua atualização dos saberes. Também pode refletir as oportunidades aproveitadas, pois o perfil exigido pelas instituições de saúde demonstra o conhecimento e aptidão valorizados em um determinado período (CARVALHO; KALINKE, 2008).

A educação propicia a transformação dos paradigmas dos indivíduos, possibilitando que este compreenda o que ocorre na sociedade, ampliando assim, a visão do mundo no qual está inserido (GUIMARÃES; MARTIN; RABELO, 2010).

A educação em serviço ocorre no ambiente de trabalho, e pode ser entendida como atualização e aprimoramento da equipe em razão das constantes mudanças científicas e tecnológicas visando o atendimento das necessidades apresentadas pelos profissionais em seus processos de trabalho (BRAGA; MELLEIRO, 2009).

A vigilância em saúde ocorre pelo diagnóstico de uma determinada situação de saúde, que irá gerar ações de acompanhamento terapêutico aos agravos que já estão instalados, determinação dos aspectos que requerem atenção, a definição de quem, como e quando se deve vigiar e, ao mesmo tempo, a definição das estratégias a serem tomadas para minimizar ou evitar a ocorrência (FRACOLLI et al., 2008).

Nos cargos dentro da estrutura do Serviço de Enfermagem a enfermeira administrativa assume o gerenciamento das unidades e a coordenação das atividades assistenciais que são realizadas pelo conjunto da equipe de saúde. Assim, como citam Castilho e Gonçalves (2005), ela toma sob sua responsabilidade a organização desses recursos com a finalidade de facilitar a assistência de enfermagem.

Ela também assume o papel de mediadora em espaços de tensão entre funcionários, membros da equipe, pacientes e familiares. Conforme Bocatto (2009), cabe à enfermeira identificar o tipo de conflito, seus estágios e usar estratégias para administrar a situação conforme se apresenta, para que o conflito se converta em oportunidade de crescimento e desenvolvimento da equipe.

O trabalho administrativo da enfermeira exige que ela atenda as expectativas relacionadas às diversas dimensões que permeiam esta atividade, exercendo papéis e funções interligados, de modo a fazer a interlocução com outros departamentos e com sua própria unidade de trabalho, garantindo o funcionamento dos serviços de saúde.

Nas atividades assistenciais as enfermeiras enfatizam o planejamento dos cuidados diários de enfermagem, o cuidado direto ao paciente e a execução de cuidados de enfermagem de maior complexidade técnica. A enfermeira tem como objeto de intervenção as necessidades de cuidado de enfermagem, que se dá através da articulação entre a ação de planejamento da assistência e a ação de cuidado direto acompanhado da interação e do vínculo com o paciente.

Nas atividades assistenciais, a enfermeira é qualificada profissionalmente e capacitada legalmente para desenvolver o cuidado integral aos pacientes (BRASIL, 1986). O procedimento técnico atrelado ao planejamento de ações, garante 
ao paciente o cuidado de que ele necessita, pois, ao planejar, a enfermeira determina os objetivos a serem alcançados para promover assistência de enfermagem de forma a adequar-se às necessidades dos pacientes (HAUSMANN; PEDUZZI, 2009).

A educação em serviço ocorre no ambiente de trabalho e compreende a atualização e aprimoramento da equipe em razão das constantes mudanças científicas e tecnológicas, visando o atendimento das necessidades apresentadas pelos profissionais no desenvolvimento de seus processos de trabalho (BRAGA; MELLEIRO, 2009). Caracterizada como um processo, a ação educativa compõe o processo de trabalho da enfermeira e deve estar incorporada no seu cotidiano.

Entretanto, para as enfermeiras do estudo, a educação em serviço não faz parte das suas atividades cotidianas, mesmo considerando o fato de ser um processo educativo a ser trabalhado com o intento de desenvolver capacidades cognitivas, psicomotoras e relacionais dos profissionais, assim como seu aperfeiçoamento diante da evolução científica e tecnológica. Desta maneira, a educação em serviço eleva a competência e valorização profissional e institucional (PASCHOAL; MANTOVANI; MÉIER, 2007).

A função educativa da enfermeira é estendida aos pacientes e acompanhantes, e consiste em educar e ensinar sobre a saúde e a doença. Ela é a combinação de aprendizagem planejada, destinadas a facilitar a adoção voluntária de comportamentos que conduzem à saúde. A enfermeira ensina sobre a saúde e auxilia as pessoas a tomarem suas decisões a partir do que foi ensinado (DALLAIRE; DALLAIRE, 2008).

No acompanhamento de acadêmicos e estagiários, uma vez que a instituição é um hospital escola, a enfermeira cumpre um papel fundamental no processo de aprendizagem do aluno que desenvolve o estágio curricular em sua unidade de trabalho, influenciando no desenvolvimento de suas habilidades, técnicas e atitudes. As condições concretas do campo de trabalho possibilitam que o estagiário vivencie situações reais. Ela é uma referência importante de trabalho, participando como facilitadora e integradora do aluno ao serviço, a equipe multiprofissional de saúde, familiares e usuários (ITO; TAKAHASHI, 2005).

A pesquisa desenvolvida pela enfermeira, juntamente com as funções assistencial, gerencial e educativa, interligada no fazer cotidiano, possibilita e dá visibilidade à sua participação no processo de construção do saber, pois é através dela que a realidade é vista de forma crítica e possibilita a construção de processos e produtos específicos ligados à enfermagem (DALLAIRE; DALLAIRE, 2008). Considerando o tempo de formação dos participantes, constata-se que a realização de pesquisa não faz parte do dia a dia das enfermeiras, considerada como uma atividade de difícil realização.

Observa-se pelas declarações das enfermeiras que elas estão ligadas mais ao desempenho das funções tradicionais da enfermagem, ou seja, ao conjunto de atividades relacionadas ao cuidado, ao gerenciamento do cuidado e da unidade, mas trabalham pouco a educação e a pesquisa.

Ocorreu uma adaptação dos cargos tradicionais de enfermagem em cargos supostamente mais aderentes ao modelo gerencial vigente, alguns com mudanças significativas nas suas atribuições, como a Direção de Enfermagem que foi substituída por uma Coordenação, as antigas Coordenações de Enfermagem porárea transformam-se em Supervisão de Enfermagem por Unidade Gerencial. Houve a criação do cargo de Assessoria de Enfermagem da Direção de Assistência que agrupa várias das atividades da antiga Direção de Enfermagem.

A enfermeira saiu do gerenciamento de uma unidade para gerenciar a assistência da instituição, com todas as implicações sociais e políticas que o cargo traz embutido. Esta função proporciona um status que cargos de enfermagem não são capazes de dar. 
Em contrapartida, com a migração das enfermeiras para outros cargos, a assistência ficou descoberta, situação que fragiliza o cuidado, já que não foram conquistadas novas vagas de enfermeiras para reposição. Neste caso, percebe-se que a lógica da assistência centrada no usuário, característica do modelo gerencial atual, é menos valorizada, na medida em que dá prioridade a outros fatores.

Para as enfermeiras que atuam em cargos gerais externos ao Serviço de Enfermagem o planejamento é imprescindível no exercício das suas atividades. Para que o cuidado ocorra com efetividade é indispensável que as ações decorram de um projeto de trabalho organizado e permanente, baseado nas políticas e diretrizes às quais a organização está vinculada, considerando as condições e os meios de que dispõe, com a definição de metas claras e específicas que reflitam o conjunto de aspirações institucionais em ações práticas, que permitirão alcançar os objetivos desejados (SENHORAS, 2007).

O grande desafio gerencial das UG é e o gerenciamento aberto e flexível, incorporando a dimensão comunicativa, a distribuição mais homogênea do poder e autonomia para decidir. A operacionalização deste processo de democratização da vida organizacional ocorre nos colegiados de gestão, tanto ao nível das UG, como o colegiado de gestão geral do hospital, ao agrupar os diretores, gerentes e coordenadores de todas as unidades (CECÍLIO, 2009).

\section{Considerações finais}

Considera-se que as mudanças gerenciais ocorridas no hospital a partir de 2002 expandiram as possibilidades de atuação das enfermeiras, pois elas passaram a ocupar cargos externos à estrutura do Serviço de Enfermagem, cargos que até antes da implantação do modelo de Unidades Gerenciais, lhes eram tradicionalmente inacessíveis. É o caso da Direção da Assistência que pela primeira vez na história do hospital é exercida por uma enfermeira, e das gerências de UG.

Esta ampliação de atuação pode estar relacionada aos conhecimentos de administração que a enfermeira adquire na sua formação e ao longo da prática profissional, que lhe confere uma capacitação diferenciada de outros profissionais da equipe de saúde, no que concerne ao gerenciamento em saúde.

As funções externas ao serviço de enfermagem, com as implicações sociais e políticas que trazem embutidas, proporcionam um status que cargos de enfermagem não são capazes de dar. Mas, percebese que a assistência ao paciente foi menosprezada, situação que fragiliza o cuidado.

\section{Referências}

BARDIN, L. Análise de conteúdo. Lisboa: Edições 70, 2011.

BOCCATO, S. B. G. Como gerenciar um grupo em conflito. In: MALAGUTTI, W.; CAETANO, C. C. Gestão do serviço de enfermagem no mundo globalizado. Rio de Janeiro: Rubio, 2009. p. 85-100.

BRAGA, A. T.; MELLEIRO, M. M. Percepção da equipe de enfermagem acerca de um serviço de educação continuada de um hospital universitário. Revista da Escola de Enfermagem da USP, São Paulo, v. 43, n. 2, p. 1216-20, 2009.

BRASIL. Lei $\mathrm{n}^{\circ}$ 7.498, de 25 de Junho de 1986. Dispõe sobre a regulamentação do exercício da Enfermagem e dá outras providências. Disponível em: <http://www.planalto.gov.br/ccivil_03/leis/ 17498.htm>. Acesso em: 10 set. 2009.

CARVAlHO, D. R.; KAlinKe, L. P. Perfil do enfermeiro quanto à motivação profissional e suas necessidades de desenvolvimento. Boletim de Enfermagem, Curitiba, v. 2, n. 1, p. 82-95, 2008.

CASTILHO, V.; GONÇALVES, V. L. M. Gerenciamento de recursos materiais. In: KURCGANT, P. Gerenciamento em enfermagem. São Paulo: Guanabara Koogan, 2005. p. 157-170. 
CECÍLIO, L. C. O. Mudar modelos de gestão para mudar o hospital: cadeia de apostas e engenharia de consensos. Disponível em: <http://www.saude. ms.gov.br/controle/ShowFile.php?id=19946>. Acesso em: 10 set. 2009.

Cecílio, L. C. O.; Merhy, E. E. Integralidade do cuidado como eixo da gestão hospitalar. 2003. Disponível em: <http://www.hc.ufmg.br/gids/ Integralidade.pdf $>$. Acesso em: 10 set. 2009.

DALLAIRE, C.; DALLAIRE, M. Le savoir infirmier dans les fonctions infirmières. In: DALLAIRE, C. Le savoir infirmier: au couer de la discipline et de la profession. Montréal: Gaëtan Morin, 2008. p. 265305.

FRACOLLI, L. A.; FARIA, L. S.; PEREIRA, J. G.; MELCHIOR, R.; BERTOLOZZI, M. R. Vigilância à Saúde: deve se constituir como política pública? Saúde e Sociedade, São Paulo, v. 17, n. 2, p. 184192, 2008.

GUIMARÃES, E. M. P.; MARTIN, S. H.; RABELO, F. C. P. Educação permanente em saúde: reflexões e desafios. Ciencia y Enfermería, Concepción, v. 16, n. 3, p. 25-33, 2010.

HAUSMANN, M.; PEDUZZI, M. Articulação entre as dimensões gerencial e assistencial do processo de trabalho do enfermeiro. Texto \& Contexto Enfermagem, Florianópolis, v. 18, n. 2, p. 258-65, 2009.

ITO, E. E.; TAKAHASHI, R. T. Percepções dos enfermeiros de campo sobre o estágio curricular da graduação de enfermagem realizados em sua unidade de trabalho. Revista da Escola de Enfermagem da USP, São Paulo, v. 39, n. 1, p. 109-110, 2005.

MINAYO, M. C. S.; DESLANDES, S. F.; GOMES, R. (Org.). Pesquisa social: teoria, método e criatividade. 27. ed. Petrópolis: Vozes, 2008.

PAGANINI, M. C.; GRESPAN SUAREZ, G.; SEIFFERT, L.; PEDROZO, M. H.; ALBINI, R. M. N.; WALACH, V.R. Oficinas sobre papéis e funções de enfermeiras em hospital universitário - relato de experiência. Cogitare Enfermagem, Curitiba, v. 5, p. 16-24, 2000.
PASCHOAL, A. S.; MANTOVANI, M. de F.; MÉIER, M. J. Percepção da educação permanente, continuada e em serviço para enfermeiros de um hospital de ensino. Revista da Escola de Enfermagem da USP, São Paulo, v. 41, n. 3, p. 478-84, 2007.

SENHORAS, E. M. A cultura na organização hospitalar e as políticas culturais de coordenação de comunicação e aprendizagem. Revista Eletrônica de Comunicação \& Inovação em Saúde Fio Cruz, Rio de Janeiro, v. 1, n. 1, p. 45-55, 2007.

UNIVERSIDADE FEDERAL DO PARANÁ. Departamento de Pessoal. Relação de funcionários do Hospital de Clínicas/UFPR. Curitiba: $\mathrm{HC} /$ UFPR, 2011.

- Hospital de Clínicas. Assessoria de Planejamento. Coletânea sobre as unidades funcionais da UFPR. Curitiba: HC/UFPR, 2002.
Recebido em: 27 jun. 2014 Aceito em: $18 \mathrm{fev} .2015$. 
